\title{
Corrigendum
}

\section{Restoration of gait with orthoses in thoracic paraplegia: a multicentric investigation}

S Lotta, A Fiocchi, R Giovannini, R Silvestrin, L Tesio, A Raschi, L Macchia, V Chiapatti, M Zambelli, C Tosi, S Baratta, M Franceschini Paraplegia 1994; 32: 608-615

Please note that in the above paper all the Parawalker patients used crutches NOT walking frames as indicated in the text. 